\title{
„Kochsalz verbieten?“ - ein nachdenkliches Vorwort zur Beitragsserie Regulatorische Ökotoxikologie
}

\author{
Tobias Frische $\cdot$ Jan Ahlers $\cdot$ Bettina Hitzfeld (Herausgeber und Herausgeberin)
}

Erhalten: 24. Oktober 2008/Akzeptiert: 12. Dezember 2008/Online veröffentlicht: 14. Januar 2009

(C) Springer-Verlag 2009

Zusammenfassung Ziel der Beitragsserie ,regulatorische Ökotoxikologie“ ist es, (i) die gesetzlichen Grundlagen, Prinzipien und Instrumente der ökotoxikologischen Stoffund Risikobewertung zu erläutern, (ii) die Denk- und Arbeitsweisen von Regulatoren zu beschreiben, (iii) die Abstimmungs- und Entscheidungsprozesse in der Stoff- und Risikobewertung nachzuzeichnen, (iv) unterschiedliche Standpunkte zu strittigen regulatorischen Themen zu vermitteln und (v) aktuelle Entwicklungen in der regulatorischen Ökotoxikologie aufzuzeigen. Die Serie umfasst Diskussionsbeiträge und Originalarbeiten von Autorinnen und Autoren aus den maßgeblich beteiligten Interessensgruppen (Behörde, Industrie, Akademie, Umweltverbände), wobei mit unterschiedlichen Fragestellungen sämtliche relevanten Stoffgruppen, Methoden und stoffgesetzlichen Regelungsbereiche (Biozide, Pflanzenschutzmittel, Arzneimittel, Abwasserreinigung, REACH, Wasserrahmenrichtlinie) adressiert werden.

Schlüsselwörter Chemikalienbewertung $\cdot$ Regulatorische Ökotoxikologie $\cdot$ Risikokommunikation $\cdot$ Stoff- und Umweltgesetzgebung · Umweltrisikobewertung

T. Frische

Umweltbundesamt (UBA), Fachgebiet Pflanzenschutzmittel,

Wörlitzer Platz 1, 06844 Dessau, Deutschland

E-Mail: tobias.frische@uba.de

\section{J. Ahlers $(\square)$}

Umweltbundesamt (UBA),

Ahrenshooper Zeile 1A, 14129 Berlin, Deutschland

E-Mail: jan.ahlers@t-online.de

\section{B. Hitzfeld}

Bundesamt für Umwelt, Abt. Stoffe, Boden, Biotechnologie, 3003 Bern, Schweiz

E-Mail: bettina.hitzfeld@bafu.admin.ch

\begin{abstract}
A ban on table salt? - a reflective preamble to the series of articles on regulatory ecotoxicology
\end{abstract}

\begin{abstract}
This series of articles titled "Regulatory Ecotoxicology" is intended to (i) elucidate the legal background, general principles and instruments of ecotoxicological risk assessment of chemicals, (ii) exemplify the thinking and functioning of regulatory decision making, (iii) illustrate the consulting and decision making processes in risk assessment of chemicals, (iv) communicate different positions on controversial regulatory topics and (v) highlight recent trends in regulatory ecotoxicology. Both discussion papers and scientific articles are contributed by authors representing all stakeholders involved (administrative bodies, industry, academia, environmental associations). The issues addressed cover all relevant substance groups, methods and regulatory instruments (biocides, plant protection products, pharmaceuticals, wastewater treatment, REACH, Water Framework Directive).
\end{abstract}

Keywords Chemical risk assessment - Environmental legislation · Environmental risk assessment ·

Legislation of chemicals · Regulatory ecotoxicology · Risk communication

\section{Problemstellung}

Der Nutzen chemischer Stoffe ist in unseren industriell geprägten Gesellschaften weitestgehend unstrittig. Und dennoch wird regelmäßig und oftmals heftig über Chemikalien gestritten. Streitpunkt sind zumeist unerwünschte Stoffeigenschaften, die eine Gefahr für die menschliche Gesundheit und die Umwelt darstellen können und/oder die mit der Nutzung (potenziell) gesundheits- und umweltgefährli- 
cher Chemikalien einhergehenden Risiken. Dieser Streit ist grundsätzlich nichts Negatives. Im Gegenteil: Kontroverser öffentlicher Diskurs ist der beste Indikator für eine lebendige Demokratie, ist der Motor für öffentliche Meinungsbildung und politische Entscheidungsfindung und damit für gesellschaftlichen Fortschritt. Soweit die Theorie. Doch wie sieht es in der Praxis aus?

Die Umweltorganisation Greenpeace hat im Juni 2008 eine Studie „The Dirty Portfolios of the Pesticides Industry“ veröffentlicht (www.greenpeace.de). In der Studie werden viele Pflanzenschutzmittel, die von den im Industrieverband Agrar (IVA) organisierten Firmen vermarktet werden, nach von Greenpeace definierten Kriterien als ,,besonders gefährlich“ für Mensch und Umwelt eingestuft. Darauf konterte der Industrieverband mit einer Pressemitteilung, in der es hieß: „Nach den Maßstäben von Greenpeace müsste allerdings auch Kochsalz verboten werden." Gegen diese Aussage wehrte sich die Umweltorganisation erfolgreich vor dem Landgericht Frankfurt/Main. Mit dessen Beschluss vom 30. Juli 2008 wurde der IVA zu einer Gegendarstellung verpflichtet, in der richtig gestellt wird: „Nach den Maßstäben von Greenpeace müsste Kochsalz jedoch keinesfalls verboten bzw. auf die aus den Untersuchungen abgeleitete ,Schwarze Liste“ gesetzt werden.“ (www.iva.de).

\section{2 Ökotoxikologie im regulatorischen Kontext - ein komplexer Entscheidungsfindungsprozess}

Aus fachlicher Sicht ist dieses öffentliche Scharmützel zum Schmunzeln. Oder eher nicht? Sicherlich vermögen Stilmittel wie Bagatellisierung oder Skandalisierung zähe Debatten anzufeuern. Zielführend ist ein Streit auf einem solch bizarren Niveau jedoch mitnichten; zumindest dann nicht, wenn das Ziel eine transparente, konstruktive und fachlich solide Debatte sein soll. Ein derartiges Ziel wird aber offensichtlich eher selten angestrebt - und das ist ärgerlich, weil in der Öffentlichkeit ein grundlegend falscher Eindruck davon erweckt wird, was Stoff- und Risikobewertung zum Schutz von Mensch und Umwelt tatsächlich bedeuten: Ein komplexer Entscheidungsfindungsprozess im Spannungsfeld von wissenschaftlichem Anspruch sowie wirtschaftlichen und politischen Interessen. Hier genau ist sie gefordert, die Ökotoxikologie im regulatorischen Kontext. Wesentliche Aufgaben dieser Disziplin sind es, die Entwicklung verlässlicher Instrumente zur Erzeugung entscheidungsrelevanter Faktendaten (Test- und Analysemethoden, Modelle, usw.) voranzutreiben und Bewertungsstrategien zu etablieren, welche auf der Basis umfangreicher und oft komplexer Informationen fundierte und nachvollziehbare Entscheidungen zu Gefahren und Risiken von Chemikalien ermöglichen. Um den wissenschaftlichen Anspruch einzulösen, ist eine kontinuierliche Integration des Standes von (forschender) Wissenschaft und Technik in die Entscheidungsprozesse erforderlich. Gleichzeitig entwickeln oder ändern sich gesellschaftliche Wertvorstellungen und politische Ziele, nationale und internationale Gesetzgebungen, Zuständigkeiten von Behörden und Institutionen sowie Aufgaben und Pflichten für Produzenten und Anwender von Chemikalien. Hinzu kommt, dass sich diese Prozesse mit den Möglichkeiten des Informationszeitalters kontinuierlich beschleunigen.

Die zunehmende Komplexität in der Stoff- und Risikobewertung birgt - abgesehen von dem mehr oder weniger bewussten Rückgriff auf allzu schlichte oder unsachliche Argumente - eine weitere ernsthafte Gefahr: Mangelnde Transparenz und schwindende Beteiligung der (Fach-)Öffentlichkeit an der Diskussion um Gefahren und Risiken von Chemikalien. Beiden Tendenzen gilt es, durch eine angemessene Beteiligungs- und Streitkultur entgegenzuwirken. Was ist also von den beteiligten Interessensgruppen zu fordern, damit ein niveauvoller Streit möglich ist? Von den Umwelt- und Verbraucherverbänden ist zu fordern, dass sie ihre Kritik und Alternativvorschläge zur Stoff- und Risikobewertung - unter Diskussion der Unterschiede zur derzeitigen, per Gesetz definierten Bewertungspraxis fachlich besser und nachvollziehbarer begründen. Die Industrie müsste ihre übliche Verteidigungshaltung aufgeben und sich um eine differenziertere inhaltliche Diskussion über den Nutzen und die Risiken ihrer chemischen Produkte bemühen. Die berechtigten Forderungen der Gesellschaft nach verstärkter Vorsorgeorientierung und Nachhaltigkeit im Umgang mit (potenziell) gesundheits- und umweltgefährlichen Chemikalien erfordern überzeugende Argumente und wachsendes Verantwortungsbewusstsein von Seiten der Industrie. Langfristig wird nur derjenige Produzent dauerhaften ökonomischen Erfolg haben, der die Zeichen der Zeit rechtzeitig erkannt hat und Produkte anbieten kann, die in einer Gesellschaft mit kritischem Bewusstsein für Fragen des Verbraucher- und Umweltschutzes Akzeptanz erfahren. Von den Entscheidungsinstanzen und Behörden schließlich sind stärkere Bemühungen um ein ,public understanding of regulatory decision making“ zu fordern - kurz: Transparenz, Offenheit und Diskussionsbereitschaft.

\section{Die Beitragsserie „Regulatorische Ökotoxikologie“}

Ziel der mit dieser Ausgabe beginnenden Beitragsserie „Regulatorische Ökotoxikologie" ist es daher, insbesondere den nicht oder nur indirekt mit regulatorischen Fragen beschäftigten Leserinnen und Lesern (i) die gesetzlichen Grundlagen, Prinzipien und Instrumente der ökotoxikologischen Stoff- und Risikobewertung zu erläutern, (ii) die Denk- und Arbeitsweisen von Regulatoren näher zu bringen, (iii) die Abstimmungs- und Entscheidungsprozesse in der Stoff- 
und Risikobewertung nachzuzeichnen, (iv) unterschiedliche Standpunkte zu strittigen regulatorischen Themen zu vermitteln und (v) aktuelle Entwicklungen in der regulatorischen Ökotoxikologie aufzuzeigen. Verbunden hiermit ist der Wunsch, dass besonders bei den Kolleginnen und Kollegen in der akademischen Umweltforschung das Verständnis für relevante regulatorische Fragestellungen geschärft und das Interesse an einer aktiven Beteiligung an der Diskussion um adäquate Entscheidungen gefördert wird.

Vorgesehen sind Beiträge, die sich den Kategorien „Diskussionsbeiträge“ und „Originalarbeiten“ zuordnen lassen. Diskussionsbeiträge erlauben Autoren aus den beteiligten Interessensgruppen (Behörde, Industrie, Akademien, Umweltverbände) fachliche Argumente und Stellungnahmen zu einzelnen Themen aus ihrer jeweiligen Sichtweise zu formulieren. Sofern entsprechende Kurzbeiträge mehrerer Interessensgruppen vorliegen, werden diese nach Möglichkeit in derselben Ausgabe platziert. Die Qualitätssicherung der Diskussionsbeiträge erfolgt wegen ihres von wissenschaftlichen Originalarbeiten abweichenden Charakters nicht durch ein Peer-Review mit externen Gutachtern, sondern wird von den Herausgebern und der Herausgeberin der Serie vorgenommen. Dabei geht es ausschließlich um eine kritische Durchsicht hinsichtlich verfälschender Darstellungen sowie grober Fehler. Bei Originalarbeiten nimmt die Dokumentation von Forschungsergebnissen einen höheren Anteil ein, weshalb für diese Beiträge ein reguläres Begutachtungsverfahren vorgesehen ist.

Das Themenspektrum der vorgesehenen Beiträge ist breit gefasst, was die Vielfalt der stoffgesetzlichen Regelungsbereiche widerspiegelt. Den Auftakt in dieser Ausgabe gestalten zwei Beiträge:

- Jan Ahlers und Klaus-Peter Ebke (2009) berichten über bisherige Erfahrungen mit der Durchführung des Moduls „Regulatorische Ökotoxikologie“ im gemeinsam von SETAC-GLB und GDCh getragenen Postgradualstudium „Fachökotoxikologie“.

- Ev Kretschmar et al. (2009) reflektieren die bisherigen Praxiserfahrungen mit der noch relativ jungen Umweltrisikobewertung von Bioziden und geben einen Ausblick auf zukünftige Entwicklungen.

Im Anschluss an den letztgenannten Beitrag wird in dieser Ausgabe ein Dreiteiler zum Thema „Wege zur Beurteilung der Umweltverträglichkeit biozider Wirkstoffe" publiziert, herausgegeben von Walter Giger (Bürgi et al. 2009; Buser u. Morf 2009; Burkhardt et al. 2009). Diese drei Beiträge, zusammen mit einem Vorwort von Walter Giger et al. (2009), erscheinen in der Rubrik „Regulatorische Ökotoxikologie und Stoffbewertung“, was einerseits die Nähe zu der hier vorgestellten Beitragsserie aufzeigt, andererseits aber dokumentiert, dass es sich um drei bzw. vier eigenständige Publikationen handelt, die parallel zur Beitragsserie „Regulatorische Ökotoxikologie" entstanden sind.
Für die Fortsetzung der Serie sind folgende Themen bereits vorgesehen:

- „Umweltrisikobewertung von Zytostatika - Eine Herausforderung für Wissenschaft, Industrie und Regulatorik" von Ina Ebert, Armin Schuster und Klaus Kümmerer.

- „Kupferhaltige Pflanzenschutzmittel: Umweltproblematik und politische Perspektiven" von Tobias Frische, Eva Dressler, Sabine Gärtner und Jörn Wogram.

- „Anwendung ökotoxikologischer Methoden zur Bewertung weitergehender Verfahren in der Abwasserreinigung" von Hansruedi Siegrist, Michael Schärer, Bettina Hitzfeld.

- „Regulatorische Bewertung von Chemikaliengemischen unter der Wasserrahmenrichtlinie: Herangehensweisen, Probleme und Perspektiven“"von Thomas Backhaus und Michael Faust.

- „REACH-Autorisierung: Implikationen der Kandidatenlisten" von Christoph Schulte et al.

Einen besonderen Schwerpunkt innerhalb der Beitragsserie setzt die von Henning Friege herausgegebene Artikelserie „Neue Regelungen für Zulassung und Gebrauch von Pestiziden in der Diskussion“. In vier Aufsätzen wird hier ein Zwischenbericht zur Reform des EU-Rechts im Zusammenhang mit der Zulassung und Anwendung von Pflanzenschutzmitteln gegeben. Unterschiedliche Standpunkte zu einem Fragenkatalog im Zusammenhang mit dem Reformvorhaben werden jeweils aus Sicht einer Umweltbehörde (Dietlinde Großmann, UBA), der Hersteller von Pflanzenschutzmitteln (Hannelore Schmid, IVA) und einer Umweltorganisation (Susanne Smolka, PAN Deutschland) wiedergegeben.

Die Serie ist offen angelegt. Deshalb sind weitere Beiträge aus Sicht der (universitären) Forschung, Behörden, Umweltschutz- bzw. Verbraucherschutzorganisationen sowie aus der Industrie (einzeln und/oder gemeinsam) ausdrücklich erwünscht. Interessierte sind gebeten, ihren Vorschlag an Jan Ahlers zu richten (jan.ahlers@t-online.de).

Allen Autoren und Autorinnen gilt herzlicher Dank. Den Leserinnen und Lesern der UWSF wünschen wir eine anregende Lektüre.

\section{Literatur}

Ahlers J, Ebke KP (2009) Neue Herausforderungen in der regulatorischen Ökotoxikologie erfordern eine anspruchsvolle Ausbildung - Postgradualstudium „Ökotoxikologie“. Umweltwiss Schadst Forsch 21(1)

Bürgi D, Knechtenhofer L, Meier L, Giger W (2009) Priorisierung von bioziden Wirkstoffen aufgrund der potenziellen Gefährdung schweizerischer Oberflächengewässer. Umweltwiss Schadst Forsch 21(1)

Burkhardt M, Junghans M, Zuleeg S, Schoknecht U, Lamani X, Bester K, Vonbank R, Simmler H, Boller M (2009) Biozide in Gebäudefassaden - ökotoxikologische Effekte, Auswaschung und Belastungsabschätzung für Gewässer. Umweltwiss Schadst Forsch 21(1) 
Buser AM, Morf LS (2009) Stoffflussanalyse von quartären Ammoniumverbindungen für die Schweiz. Modellierung des Verbrauchs in bioziden Anwendungen und der Emissionen in die Umwelt. Umweltwiss Schadst Forsch 21(1)

Giger W, Bürgi D, Burkhardt M, Morf LS (2009) Wege zur Beurtei- lung der Umweltverträglichkeit biozider Wirkstoffe (Vorwort). Umweltwiss Schadst Forsch 21(1)

Kretschmar E, Müller-Knoche S, Schwarz-Schulz B, Nöh I (2009) Erfahrungen des Umweltbundesamtes mit der EU-Biozid-Richtlinie. Umweltwiss Schadst Forsch 21(1) 